EPJ Web of Conferences 28, 09004 (2012)

DOI: 10.1051/epjconf/20122809004

(C) Owned by the authors, published by EDP Sciences, 2012

\title{
Recent results on beyond the standard model Higgs boson searches from CMS
}

\author{
Alexander A. Savin, a on behalf of the CMS Collaboration \\ University of Wisconsin-Madison, 1150 University Ave., Madison WI 53706-1390
}

\begin{abstract}
Two extensions of the standard model, one that includes the seesaw mechanism of type II, and the minimal supersymmetric extention to the standard model, are studied using up to $1.6 \mathrm{fb}^{-1}$ of data collected in proton-proton collisions at $\sqrt{s}=7 \mathrm{TeV}$ with the CMS detector at the LHC.
\end{abstract}

\section{Introduction}

An observation of the doubly charged component $\Phi^{++}$(here and below charge conjugate modes are implicitly included) of the triplet scalar field predicted in the the minimal seesaw model of type II, would establish such mechanism in the most promising framework giving mass to neutrinos. This particle carries double electric charge and decays to the same charged lepton pairs $\ell_{i}^{+} \ell_{j}^{+}$allowing also lepton flavor violating decays. The $\Phi^{++}$Yukawa coupling matrix is proportional to the light neutrino mass matrix and allows to test the neutrino mass mechanism by measuring the branching fractions $\Phi^{++} \rightarrow \ell_{i} \ell_{j}[1]$.

The minimal supersymmetric extension to the standard model (MSSM) requires the presence of two Higgs doublets. This leads to a more complicated scalar sector, with five massive Higgs bosons: a light neutral CP-even state $(h)$, two charged states $\left(H^{ \pm}\right)$, a heavy neutral CP-even state $(H)$ and a neutral CP-odd state $(A)$.

If the charged Higgs boson mass, $m_{H^{+}}$, is smaller than the top quark mass, the top quark can decay via $t \rightarrow H^{+} b$ (and its charge conjugate). The lower limit on the charged Higgs boson mass is set to about $80 \mathrm{GeV} / c^{2}$ by LEP experiments. For values of $\tan \beta$, the ratio of the vacuum expectation values of the two Higgs boson doublets, larger than 20, the charged Higgs boson preferentially decays to $\tau$ lepton and neutrino, $H^{+} \rightarrow \tau^{+} v_{\tau}$. The presence of the $t \rightarrow H^{+} b, H^{+} \rightarrow \tau^{+} v_{\tau}$ decay modes alters the standard model (SM) prediction of the $\tau$ lepton yield in the decay products of the $t \bar{t}$ pairs. The current upper limit on the branching fraction $B R\left(t \rightarrow H^{+} b\right) \simeq 0.2$ is set by the CDF and D0 experiments at the Tevatron for $m_{H^{+}}$between 80 and $155 \mathrm{GeV} / c^{2}$, assuming $B R\left(H^{+} \rightarrow \tau^{+} v\right)=1$. The dominant source of top quarks at LHC is $p p \rightarrow t \bar{t}$ process, therefore the charged Higgs boson is searched for in the subsequent decay products of the top quark pairs: $t \bar{t} \rightarrow H^{ \pm} W^{\mp} b \bar{b}$ and $t \bar{t} \rightarrow H^{ \pm} H^{\mp} b \bar{b}$ when $H^{ \pm}$decays into $\tau$ lepton and neutrino [2].

The mass relations among the neutral MSSM Higgs bosons are such that if $m_{A} \lesssim 130 \mathrm{GeV} / c^{2}$, at large values of the parameter $\tan \beta$ the masses of the $h$ and $A$ are nearly degenerate, while that of the $H$ is approximately $130 \mathrm{GeV} / c^{2}$. If $m_{A} \gtrsim 130 \mathrm{GeV} / c^{2}$, then the masses of the $A$ and $H$ are

\footnotetext{
a e-mail: asavin@mail.cern.ch
}

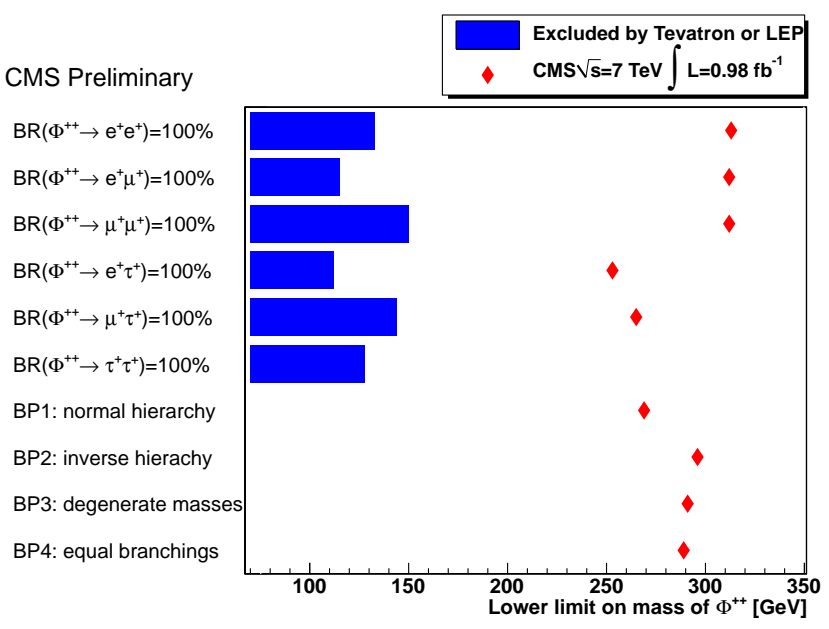

Fig. 1. Observed $\Phi^{++}$mass limits at $95 \% \mathrm{CL}$ in different lepton final states. The branching fractions that are assumed in the limit calculation are indicated.

nearly degenerate, while that of the $h$ remains near 130 $\mathrm{GeV} / \mathrm{c}^{2}$. The precise value of the crossover point depends predominantly on the nature of the mass mixing in the topsquark states. The neutral MSSM Higgs boson production is studied in its decay into pair of tau leptons, $H \rightarrow \tau \tau$, in three final states, when $\tau$ decay leptonically, one to $\mu$ and other to $e, e \mu$, and when one of the $\tau$ decays hadronically, $\mu \tau_{h}$ and $e \tau_{h}[3]$.

\section{Doubly charge Higgs boson production}

Both, the pair production process $p p \rightarrow \Phi^{++} \Phi^{--} \rightarrow \ell_{i}^{+} \ell_{j}^{+} \ell_{k}^{-} \ell_{l}^{-}$, as well as the associated production process $p p \rightarrow \Phi^{++} \Phi^{-} \rightarrow$ $\ell_{i}^{+} \ell_{j}^{+} \ell_{k}^{-} v_{l}$ are studied, assuming that the $\Phi^{++}$and $\Phi^{+}$are degenerate in mass. A search is performed for an excess of events in all possible flavour combinations of the same charge lepton pairs coming from the decays $\Phi^{++} \rightarrow \ell_{i}^{+} \ell_{j}^{+}$ without making assumptions on the $\Phi^{++}$branching fractions. Both the three and four charged lepton final states are considered including at most one and two $\tau$ leptons, respectively. The $\Phi^{++} \rightarrow W^{+} W^{+}$decays are assumed to be suppressed. 
In addition to the model independent search, the type II seesaw model is tested in four benchmark points (BP) that characterize different characteristic neutrino mass matrix structures. BP1 describes the neutrino sector with normal mass hierarchy and a massless lightest neutrino. BP2 describes the same, but with the inverse mass hierarchy. BP3 represents a degenerate neutrino mass spectrum with $m_{1}=0.2 \mathrm{eV}$. Those three benchmark points are the extremes allowed by varying the neutrino mass and hierarchy in the allowed ranges without consideration for $\theta_{13}$ or $\mathrm{CP}$ phases and cover a large region of the parameter space. The fourth benchmark point BP4 represents the case in which all $\Phi^{++}$branching fractions are equal.

The presented results are based on data corresponding to an integrated luminosity of $0.98 \mathrm{fb}^{-1}$. The final states are required to have four or three leptons with transverse momenta, $p_{\mathrm{T}}$, above $5 \mathrm{GeV} / c$ for muons and above 15 $\mathrm{GeV} / c$ for electrons and $\tau_{\mathrm{h}}$. There should be at least two leptons with $p_{\mathrm{T}}>35$ and $10 \mathrm{GeV} / c$ in the event. The $\tau_{\mathrm{h}}$ are reconstructed using "hadron plus strip"(HPS) algorithm [4] that is designed to optimize the performance of $\tau_{\mathrm{h}}$ identification and reconstruction by considering specific $\tau_{\mathrm{h}}$ decay modes. The algorithm provides high $\tau_{\mathrm{h}}$ identification efficiency, approximately $50 \%$ for the range of $\tau_{\mathrm{h}}$ energies relevant for this analysis, while keeping the misidentification rate for jets at the level of $\approx 1 \%$ that is factor of three to four times lower than for the algorithms used in the CMS physics technical design report. Detailed description of the algorithm and its performance can be found in [4].

A CLs method is used for the upper limit calculations. The results of the exclusion limit calculations are reported in Fig. 1. As can be seen, new significantly higher limits are set in comparison to the previous LEP and Tevatron bounds. The first limits on four benchmark points probe a large region of the parameter space of type II seesaw models.

\section{Study of minimal supersymmetric extension to the standard model}

\subsection{Charged Higgs boson}

The analysis is performed in three final states, $e \mu, \mu \tau_{\mathrm{h}}$ and $\tau_{\mathrm{h}} \tau_{\mathrm{h}}$, in presence of large missing transverse energy and one b-tagged jet. Transverse momenta of all leptons are required to exceed $20 \mathrm{GeV} / c, 40 \mathrm{GeV} / c$ in the $\tau_{\mathrm{h}} \tau_{\mathrm{h}}$ case.

A CLs method is used in order to obtain the upper limit at $95 \%$ CL on the excess (lack) of the events in comparison with the expected contributions from SM. The background and signal uncertainties are modeled with a lognormal probability distribution function and their correlations are taken into account. Assuming that the excess (lack) of events is due to the $t \rightarrow b H^{+}, H^{+} \rightarrow \tau^{+} v$ decays, the limit is obtained for each individual analysis, and also combined for all final states. Figure 2 shows the upper limit on $B R\left(t \rightarrow H^{+} b\right)$ assuming $B R\left(H^{+} \rightarrow \tau v\right)=1$ as a function of the higgs mass for the combination of all final states. The upper limit is set to 4-5 \% for the Higgs boson mass interval $80<m_{H^{+}}<160 \mathrm{GeV} / c^{2}$.

Figure 3 shows the exclusion region in the MSSM $M_{H^{+}}$ $\tan \beta$ parameter space obtained from the combined analysis for the MSSM $m_{h}^{\max }$ scenario: $M_{S U S Y}=1 \mathrm{TeV} / c^{2}$,

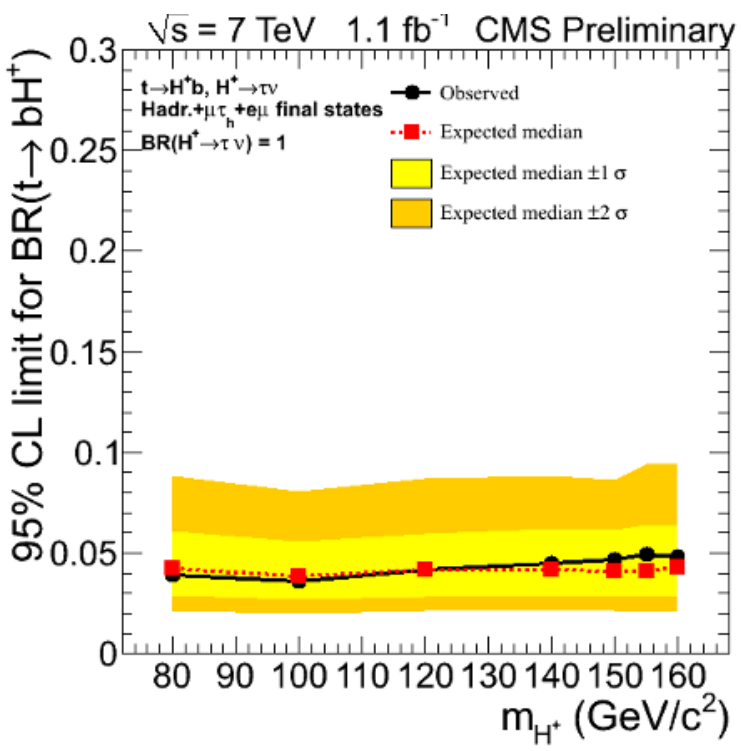

Fig. 2. Upper limit on $B R\left(t \rightarrow H^{+} b\right)$ assuming $B R\left(H^{+} \rightarrow \tau v\right)=1$ as a function of $m_{H^{+}}$for the combination of all final states. The yellow bands show the one- and two-standard-deviation ranges around the expected limit.

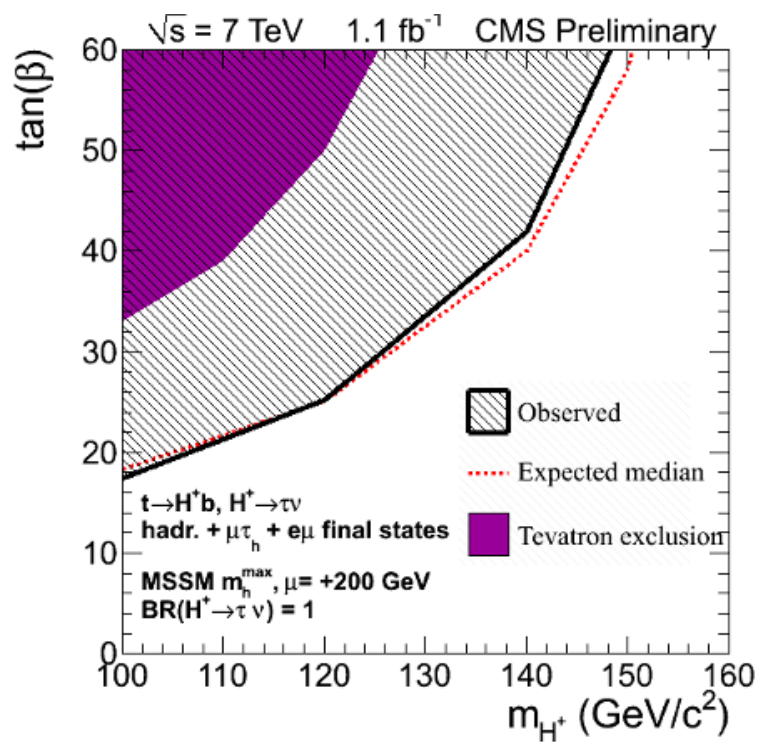

Fig. 3. The exclusion region in the MSSM $M_{H^{+}} \tan \beta$ parameter space obtained from the combined analysis for the MSSM $m_{h}^{\max }$ scenario.

$\mu=200 \mathrm{GeV} / c^{2}, M_{2}=200 \mathrm{GeV} / c^{2}, m_{\tilde{g}}=0.8 \cdot M_{S U S Y}$, $X_{t}=2 \cdot M_{S U S Y}$ (FD calculation), $X_{t}^{\overline{M S}}=\sqrt{6} \cdot M_{S U S Y}$ (RG calculation), $A_{b}=A_{t}$. $M_{\mathrm{SUSY}}$ denotes the common soft-SUSY-breaking squark mass of the third generation; $X_{t}=A_{t}-\mu / \tan \beta$ the stop mixing parameter; $A_{t}$ and $A_{b}$ the stop and sbottom trilinear couplings, respectively; $\mu$ the Higgsino mass parameter; $m_{\tilde{g}}$ the gluino mass; and $M_{2}$ the $\mathrm{SU}(2)$-gaugino mass parameter. The value of $M_{1}$ is fixed via the GUT relation $M_{1}=(5 / 3) M_{2} \sin \theta_{\mathrm{W}} / \cos \theta_{\mathrm{W}}$. In determining these bounds on $\tan \beta$, we have used the central values of the Higgs boson cross sections as a function of $\tan \beta$ reported by the LHC Higgs Cross Section Working Group. The cross sections have been obtained from the GGH@NNLO and HIGLU programs for the gluon- 
fusion process. For the $b \bar{b} \rightarrow \phi$ process, the 4-flavor calculation and the 5-flavor calculation as implemented in the BBH@NNLO program have been combined using the Santander scheme. Rescaling of the corresponding Yukawa couplings by the MSSM factors calculated with FeynHiggs has been applied. We do not quote limits above $\tan \beta=60$ as the theoretical relation between cross section and $\tan \beta$ becomes unreliable.

\subsection{Neutral Higgs boson}

The MSSM neutral Higgs boson analysis is performed in three final states $e \mu, \mu \tau_{\mathrm{h}}$, and $e \tau_{\mathrm{h}}$ with $p_{\mathrm{T}}>15 \mathrm{GeV} / c$ for muons, and $p_{\mathrm{T}}>20 \mathrm{GeV} / c$ for $e$ and $\tau_{\mathrm{h}}$. In $e \mu$ final states one of the lepton is required to have $p_{\mathrm{T}}>20 \mathrm{GeV} / c$ and other $p_{\mathrm{T}}>10 \mathrm{GeV} / c$. Each final state is subdivided into two categories, one with no b-tagged jets with $p_{\mathrm{T}}>20$ $\mathrm{GeV} / c$ and the other with at least one b-tagged jet.

The largest source of events selected with these requirements comes from the SM $Z \rightarrow \tau \tau$ production. The contribution from this process is estimated using a sample of simulated events, normalized to the number of observed $Z \rightarrow \mu \mu$ and $Z \rightarrow e e$ events in data. A significant source of background arises from QCD multijet events and $W+$ jets events in which a jet is misidentified as $\tau_{\mathrm{h}}$, and there is a real or misidentified $e$ or $\mu$. The rates for these processes are estimated using the number of observed events, where both reconstructed leptons have the same charge, samesign combination. Other background processes include $t \bar{t}$ production and $Z \rightarrow e e / \mu \mu$ events, particularly in the $e \tau_{\mathrm{h}}$ channel, due to the $2-3 \%$ probability for electrons to be misidentified as $\tau_{\mathrm{h}}$ [4]. The small fake-lepton background from $W+$ jets and QCD for the $e \mu$ channel is estimated using data.

The event generator PYTHIA, and POWHEG, and MADGRAPH are used to model the Higgs boson signal and other backgrounds. The TAUOLA package is used for tau decays in all cases.

To distinguish the Higgs boson signal from the background, we reconstruct the visible mass, defined as the invariant mass of the visible tau decay products. The observed visible mass distributions are fit in each case to the sum of the SM backgrounds and the Higgs boson signal. The visible mass spectra show no evidence for the presence of a Higgs boson signal, and are used to set a 95\% CL upper bound on the product of the Higgs boson cross section and the tau-pair branching fraction, $\sigma_{\phi} \cdot B_{\tau \tau}$.

Figure 4 shows the upper bound on $\sigma_{\phi} \cdot B_{\tau \tau}$ as a function of $m_{A}$, where we use as the signal acceptance model the combined visible mass spectra from the $g g$ and $b \bar{b}$ production processes for $h, A$, and $H$, and assuming $\tan \beta=30$. The plot also shows the one- and two-standard-deviation range of expected upper limits for various potential experimental outcomes. The observed limits are well within the expected range assuming no signal.

One can interpret the upper limits on $\sigma_{\phi} \cdot B_{\tau \tau}$ in the MSSM parameter space of $\tan \beta$ versus $m_{A}$ for an example scenario in the same way it was done above for the charged Higgs. The results are shown in Fig. 5. They exclude a region in $\tan \beta$ down to values smaller than those excluded by the Tevatron experiments for $m_{A} \lesssim 140 \mathrm{GeV} / c^{2}$, and significantly extend the excluded region of MSSM param-

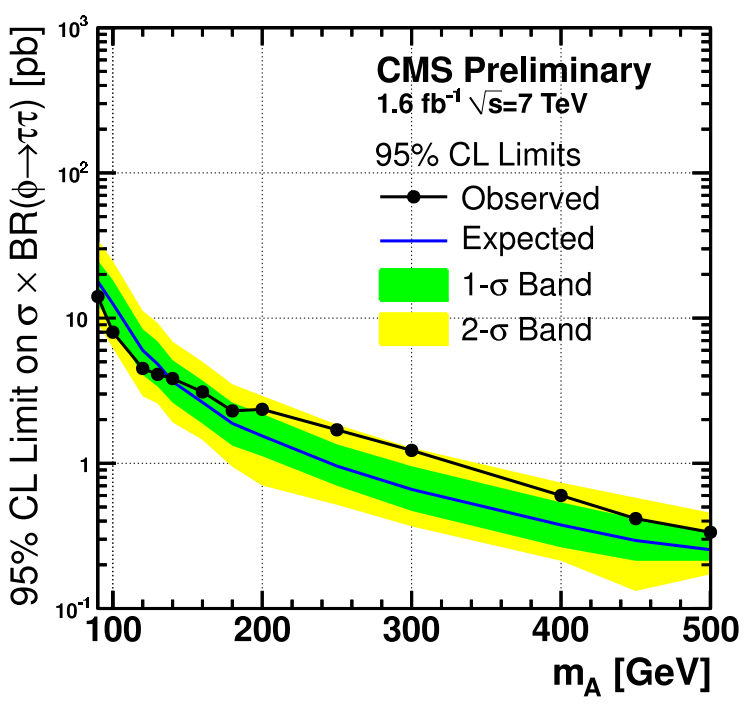

Fig. 4. The expected one- and two-standard-deviation ranges and observed $95 \%$ CL upper limits on $\sigma_{\phi} \cdot B_{\tau \tau}$ as a function of $m_{A}$. The signal acceptance is based on the MSSM model described in the text, assuming $\tan \beta=30$.

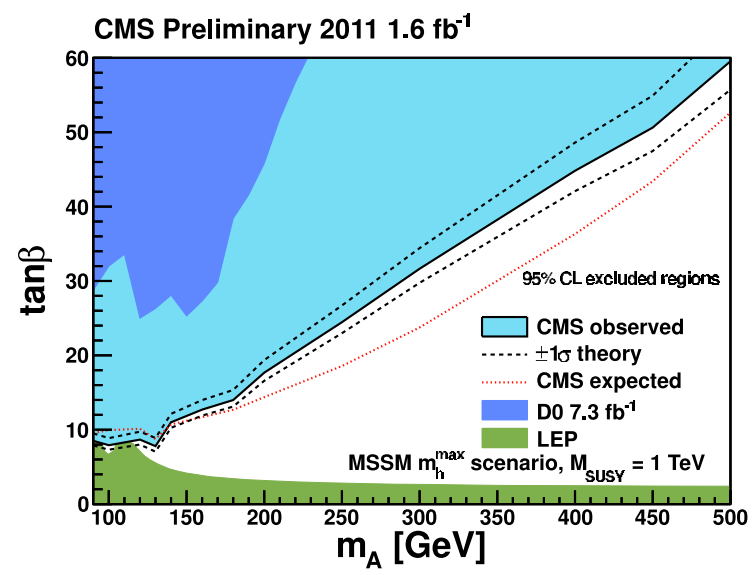

Fig. 5. Region in the parameter space of $\tan \beta$ versus $m_{A}$ excluded at $95 \% \mathrm{CL}$ in the context of the MSSM $m_{h}^{\max }$ scenario, with the effect of $\pm 1 \sigma$ theoretical uncertainties shown. The other shaded regions show the 95\% CL excluded regions from the LEP and Tevatron experiments.

eter space at larger values of $m_{A}$. Figure 5 also shows the region excluded by the LEP experiments.

\section{References}

1. CMS Collaboration, CMS-PAS-HIG-11-007, 2011, and references therein

2. CMS Collaboration, CMS-PAS-HIG-11-008, 2011, and references therein

3. CMS Collaboration, CMS-PAS-HIG-11-020, 2011, and references therein

4. CMS Collaboration, CMS-PAS-TAU-11-001, 2011, accepted by JINST 